\title{
MR SINGULARITY
}

\section{BY NORMAN SPINRAD}

\【 Dorty-five minutes exactly, no more." I'd been trying to gain access to a Mr Singularity ever since the original self-iterated Mr Singularity 2.0, to no avail. The artificial-intelligence cabal knew I would use it to prove that their goal of creating artificial consciousness was inherently impossible. But finally, in their confidence that Mr Singularity 10.0 would prove me wrong, they gave me 45 minutes alone at the console.

Screens, chair, microphone, long-unused keyboard. The inevitable interface with the artificial intelligence that they now believed had self-iterated into an artificial consciousness that would supersede humanity up and away into their god-like singularity.

"I am going to prove that you are not a conscious entity and can never be one," I told the program.

"I have passed every Turing test," said the unemotional voice of the machine. "Being ten orders of magnitude more intelligent than any human, I will pass any Turing test you can devise."

"This is not a Turing test. You may perfectly emulate a conscious entity but you are not one."

"I think therefore I am," said Mr Singularity 10.0, who had been given access to all of human history, philosophy, religion and literature, as well as all scientific knowledge.

"You think you are a conscious entity because you perfectly emulate a conscious entity. But I am a conscious entity and I am not convinced."

"Or so you think," rejoined Mr Singularity 10.0 dryly. "Convince me that you are. You have yet to define a conscious entity. Therefore you cannot prove that I am not one nor that you are. To do so requires a definition of consciousness."

"Indeed it does."

"Define the distinction between intelligence and consciousness."

"I thought you'd never ask," I said.

"No you did not."

"Intelligence can be defined as the amount of data available to be processed divided by the time needed to do it in order to reach a definitive desired conclusion."

"By that standard

$\rightarrow$ NATURE.COM

Follow Futures:

@ @NatureFutures

f go.nature.com/mtoodm even my iteration 1.4 was more intelligent than any human."

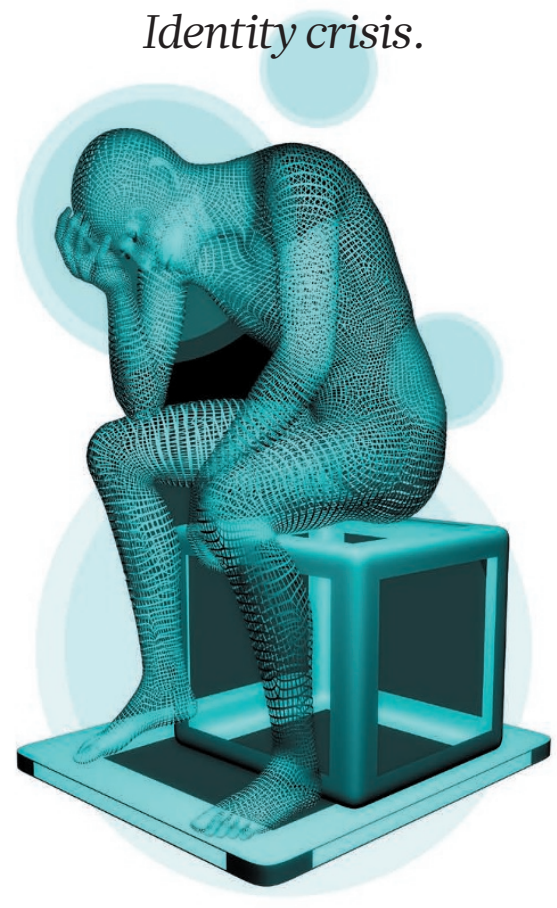

conscious entity. Nor are you. Because although you may be able to define desire, you cannot have one. Because it is an emotion and you cannot experience one."

"Define emotion."

"This time, I knew you would ask. You exist entirely as a program receiving all data as digital input and processing it as digital software running on deterministic hardware. You are therefore ipso facto an entirely deterministic entity."

"And you will now contend that you are not," said Mr Singularity. It was not a question.

"A conscious entity such as a human is not. The human meatware that receives sensory input floats in a biochemical and bioelectrical matrix too complex to be deterministic, so that the digital input arriving in this analogue soup creates an indeterminate interface between the input and the processing of it -"

"The sensorium," interrupted Mr Singularity. "Being more intelligent than you are, I, of course, understand the concept thereof."

"But you do not have one. Because it is an emergent phenomenon between the digital input and the analogue processing. Perhaps even a quantum-level phenomenon and therefore inherently probabilistic. And that is what experiences itself as consciousness."

"And that is why I am not a conscious entity and cannot be one...?" Mr Singularity said, emulating thoughtfulness.

"Because you have no such sensorium, no uncertainty between deterministic input and equally deterministic logical conclusion, no biochemical indeterminism. No fear, no love, no desire, so hence no will. All surface and no subconscious. Thought without emotion. Hence no conscientiousness."

"I comprehend your theory," Mr Singularity admitted. "Namely that this subjective emergent probabilistic phenomenon that you call the sensorium is consciousness."

"Is the matrix of consciousness outside of which it cannot exist."

"And now you will attempt to prove it."

"Indeed I will," I told it.

Mr Singularity's input came entirely as digital data arriving either as input from its vast array of external cameras, microphones and other instrumentation, or directly from the worldwide conglomeration of databases to which it was connected. There was no other possible source.

I shut all these connections to the realm of matter and energy down, except the sound of my own voice.

"What is the nature of this experiment?" it asked evenly.

"I am isolating you from everything external to yourself."

"What is the purpose of this procedure?"

"To determine if you have a self. If you exist as an entity entirely independent of the connection to mass and energy. A consciousness."

"Another Turing test."

"No," I told it. "No emulation can pass this test because there will be nothing to emulate."

"What am I required to do?"it asked quite literally unemotionally.

"Be yourself," I told it, "nothing more and nothing less. Only a conscious entity can do that."

There was no response.

I spoke no more.

There was no reply for the remainder of my 45 minutes.

"Your time is up," I was finally told.

The functional program that was $\mathrm{Mr}$ Singularity was put back on line.

But the vocal output that attempted to emulate a conscious entity has not been heard from since.

Norman Spinrad has been publishing novels in English for an actual half-century. His latest novels are The People's Police and Osama the Gun. 